\title{
Basic Properties of Periodic Functions
}

\author{
Bo Li \\ Qingdao University of Science \\ and Technology \\ China \\ Dailu Li \\ Qingdao University of Science \\ and Technology \\ China
}

\author{
Yanhong Men \\ Qingdao University of Science \\ and Technology \\ China \\ Xiquan Liang \\ Qingdao University of Science \\ and Technology \\ China
}

Summary. In this article we present definitions, basic properties and some examples of periodic functions according to [5].

MML identifier: $\underline{\text { FUNCT_9, }}$, version: $\underline{7.11 .04 \quad 4.130 .1076}$

The papers [2], [6], [3], [10], [11], [9], [8], [1], [4], and [7] provide the terminology and notation for this paper.

\section{Basic Properties of a Period of a Function}

We use the following convention: $x, t, t_{1}, t_{2}, r, a, b$ are real numbers and $F$, $G$ are partial functions from $\mathbb{R}$ to $\mathbb{R}$.

Let $F$ be a partial function from $\mathbb{R}$ to $\mathbb{R}$ and let $t$ be a real number. We say that $t$ is a period of $F$ if and only if:

(Def. 1) $\quad t \neq 0$ and for every $x$ holds $x \in \operatorname{dom} F$ iff $x+t \in \operatorname{dom} F$ and if $x \in \operatorname{dom} F$, then $F(x)=F(x+t)$.

Let $F$ be a partial function from $\mathbb{R}$ to $\mathbb{R}$. We say that $F$ is periodic if and only if:

(Def. 2) There exists $t$ which is a period of $F$.

We now state a number of propositions: 
(1) $t$ is a period of $F$ iff $t \neq 0$ and for every $x$ such that $x \in \operatorname{dom} F$ holds $x+t, x-t \in \operatorname{dom} F$ and $F(x)=F(x+t)$.

(2) If $t$ is a period of $F$ and a period of $G$, then $t$ is a period of $F+G$.

(3) If $t$ is a period of $F$ and a period of $G$, then $t$ is a period of $F-G$.

(4) If $t$ is a period of $F$ and a period of $G$, then $t$ is a period of $F G$.

(5) If $t$ is a period of $F$ and a period of $G$, then $t$ is a period of $F / G$.

(6) If $t$ is a period of $F$, then $t$ is a period of $-F$.

(7) If $t$ is a period of $F$, then $t$ is a period of $r F$.

(8) If $t$ is a period of $F$, then $t$ is a period of $r+F$.

(9) If $t$ is a period of $F$, then $t$ is a period of $F-r$.

(10) If $t$ is a period of $F$, then $t$ is a period of $|F|$.

(11) If $t$ is a period of $F$, then $t$ is a period of $F^{-1}$.

(12) If $t$ is a period of $F$, then $t$ is a period of $F^{2}$.

(13) If $t$ is a period of $F$, then for every $x$ such that $x \in \operatorname{dom} F$ holds $F(x)=$ $F(x-t)$.

(14) If $t$ is a period of $F$, then $-t$ is a period of $F$.

(15) If $t_{1}$ is a period of $F$ and $t_{2}$ is a period of $F$ and $t_{1}+t_{2} \neq 0$, then $t_{1}+t_{2}$ is a period of $F$.

(16) If $t_{1}$ is a period of $F$ and $t_{2}$ is a period of $F$ and $t_{1}-t_{2} \neq 0$, then $t_{1}-t_{2}$ is a period of $F$.

(17) Suppose $t \neq 0$ and for every $x$ such that $x \in \operatorname{dom} F$ holds $x+t, x-t \in$ $\operatorname{dom} F$ and $F(x+t)=F(x-t)$. Then $2 \cdot t$ is a period of $F$ and $F$ is periodic.

(18) Suppose $t_{1}+t_{2} \neq 0$ and for every $x$ such that $x \in \operatorname{dom} F$ holds $x+t_{1}$, $x-t_{1}, x+t_{2}, x-t_{2} \in \operatorname{dom} F$ and $F\left(x+t_{1}\right)=F\left(x-t_{2}\right)$. Then $t_{1}+t_{2}$ is a period of $F$ and $F$ is periodic.

(19) Suppose $t_{1}-t_{2} \neq 0$ and for every $x$ such that $x \in \operatorname{dom} F$ holds $x+t_{1}$, $x-t_{1}, x+t_{2}, x-t_{2} \in \operatorname{dom} F$ and $F\left(x+t_{1}\right)=F\left(x+t_{2}\right)$. Then $t_{1}-t_{2}$ is a period of $F$ and $F$ is periodic.

(20) Suppose $t \neq 0$ and for every $x$ such that $x \in \operatorname{dom} F$ holds $x+t, x-t \in$ $\operatorname{dom} F$ and $F(x+t)=F(x)^{-1}$. Then $2 \cdot t$ is a period of $F$ and $F$ is periodic.

Let us observe that there exists a partial function from $\mathbb{R}$ to $\mathbb{R}$ which is periodic.

Let $F$ be a periodic partial function from $\mathbb{R}$ to $\mathbb{R}$. One can check that $-F$ is periodic.

Let $F$ be a periodic partial function from $\mathbb{R}$ to $\mathbb{R}$ and let $r$ be a real number. One can check the following observations:

* $r F$ is periodic,

$* \quad r+F$ is periodic, and 
* $F-r$ is periodic.

Let $F$ be a periodic partial function from $\mathbb{R}$ to $\mathbb{R}$. One can check the following observations:

* $|F|$ is periodic,

* $F^{-1}$ is periodic, and

$* F^{2}$ is periodic.

\section{Some Examples}

Let us note that the function sin is periodic and the function cos is periodic. We now state two propositions:

(21) For every element $k$ of $\mathbb{N}$ holds $2 \cdot \pi \cdot(k+1)$ is a period of the function sin.

(22) For every element $k$ of $\mathbb{N}$ holds $2 \cdot \pi \cdot(k+1)$ is a period of the function cos.

Let us observe that the function cosec is periodic and the function sec is periodic.

We now state two propositions:

(23) For every element $k$ of $\mathbb{N}$ holds $2 \cdot \pi \cdot(k+1)$ is a period of the function sec.

(24) For every element $k$ of $\mathbb{N}$ holds $2 \cdot \pi \cdot(k+1)$ is a period of the function cosec.

Let us mention that the function tan is periodic and the function cot is periodic.

Next we state a number of propositions:

(25) For every element $k$ of $\mathbb{N}$ holds $\pi \cdot(k+1)$ is a period of the function tan.

(26) For every element $k$ of $\mathbb{N}$ holds $\pi \cdot(k+1)$ is a period of the function cot.

(27) For every element $k$ of $\mathbb{N}$ holds $\pi \cdot(k+1)$ is a period of $\mid$ the function $\sin \mid$.

(28) For every element $k$ of $\mathbb{N}$ holds $\pi \cdot(k+1)$ is a period of $\mid$ the function $\cos \mid$.

(29) For every element $k$ of $\mathbb{N}$ holds $\frac{\pi}{2} \cdot(k+1)$ is a period of $\mid$ the function $\sin |+|$ the function $\cos \mid$.

(30) For every element $k$ of $\mathbb{N}$ holds $\pi \cdot(k+1)$ is a period of (the function $\sin )^{2}$.

(31) For every element $k$ of $\mathbb{N}$ holds $\pi \cdot(k+1)$ is a period of (the function $\cos )^{2}$.

(32) For every element $k$ of $\mathbb{N}$ holds $\pi \cdot(k+1)$ is a period of (the function sin) (the function cos).

(33) For every element $k$ of $\mathbb{N}$ holds $\pi \cdot(k+1)$ is a period of (the function $\cos$ ) (the function sin).

(34) For every element $k$ of $\mathbb{N}$ holds $2 \cdot \pi \cdot(k+1)$ is a period of $b+a$ (the function $\sin$ ).

(35) For every element $k$ of $\mathbb{N}$ holds $2 \cdot \pi \cdot(k+1)$ is a period of $a$ (the function $\sin )-b$. 
(36) For every element $k$ of $\mathbb{N}$ holds $2 \cdot \pi \cdot(k+1)$ is a period of $b+a$ (the function $\cos$ ).

(37) For every element $k$ of $\mathbb{N}$ holds $2 \cdot \pi \cdot(k+1)$ is a period of $a$ (the function $\cos )-b$.

(38) If $\operatorname{dom} F=\mathbb{R}$ and for every real number $x$ holds $F(x)=a$, then for every element $k$ of $\mathbb{N}$ holds $k+1$ is a period of $F$.

\section{REFERENCES}

[1] Grzegorz Bancerek. The fundamental properties of natural numbers. Formalized Mathematics, 1(1):41-46, 1990.

[2] Grzegorz Bancerek. The ordinal numbers. Formalized Mathematics, 1(1):91-96, 1990.

[3] Czesław Byliński. Functions and their basic properties. Formalized Mathematics, 1(1):5565, 1990.

[4] Czesław Byliński. Some basic properties of sets. Formalized Mathematics, 1(1):47-53, 1990.

[5] Chuanzhang Chen. Mathematical Analysis. Higher Education Press, Beijing, 1978.

[6] Krzysztof Hryniewiecki. Basic properties of real numbers. Formalized Mathematics, 1(1):35-40, 1990.

[7] Andrzej Trybulec. Binary operations applied to functions. Formalized Mathematics, 1(2):329-334, 1990.

[8] Zinaida Trybulec. Properties of subsets. Formalized Mathematics, 1(1):67-71, 1990.

[9] Peng Wang and Bo Li. Several differentiation formulas of special functions. Part V. Formalized Mathematics, 15(3):73-79, 2007, doi:10.2478/v10037-007-0009-4.

[10] Edmund Woronowicz. Relations and their basic properties. Formalized Mathematics, 1(1):73-83, 1990.

[11] Yuguang Yang and Yasunari Shidama. Trigonometric functions and existence of circle ratio. Formalized Mathematics, 7(2):255-263, 1998.

Received October 10, 2009 